\title{
Using Optogenetics to Dissect the Neural Circuits Underlying OCD and Related Disorders
}

\author{
Sean C. Piantadosi, $B A^{1,2}$ \\ Susanne E. Ahmari, MD, PhD ${ }^{1,2,3, *}$
}

\author{
Address \\ *,1Department of Psychiatry, University of Pittsburgh, 450 Technology Drive, \\ Room 227, Pittsburgh, PA 15219, USA \\ Email: ahmarise@upmc.edu \\ ${ }^{2}$ Center for Neuroscience, University of Pittsburgh, Pittsburgh, PA, USA \\ ${ }^{3}$ Center for the Neural Basis of Cognition, Pittsburgh, PA, USA
}

Published online: 15 July 2015

(C) Springer International Publishing AG 2015

\section{This article is part of the Topical Collection on Anxiety, Obsessive Compulsive} and Related Disorders

Keywords OCD · Optogenetics - Goal-directed behavior - Habitual behavior - Ventromedial striatum - Corticostriatal . Orbitofrontal cortex $\cdot$ Dorsomedial striatum $\cdot$ Dorsolateral striatum $\cdot$ Grooming

\section{Opinion Statement}

Clinical and preclinical studies have uncovered substantial evidence that dysfunction in cortico-striatal-thalamo-cortical (CSTC) loops central to the selection of action strategies may underlie obsessive compulsive disorder $(O C D)$ symptoms. In human $O C D$, data suggest that the balance between selections of habitual versus goal-directed action strategies is disrupted, with concomitant hyperactivation of CSTC regions associated with these strategies. Preclinical lesion and inactivation studies of homologous CSTC regions in rodents have shed light on how sub-regions of the frontal cortex and striatum can have dissociable effects on the exhibition of goal-directed or habitual behavior. However, these traditional methods lack the precision necessary to dissect the exact projections and cell types underlying these behaviors. It is essential to uncover this information to begin to determine how disruption in these circuits may lead to disease pathology. Here, we summarize several recent studies that utilize optogenetics, a technique that allows stimulation or inhibition of specific neural projections and cell types using light, to further understand the contribution of CSTC activity to both action selection and the OCD-relevant behavior of perseverative grooming. Based on these experiments and findings in human $O C D$ patients, we argue that $O C D$ symptoms may not only be associated with an enhancement of habitual behavior, but also with aberrant recruitment of goal-directed neural circuits. We also discuss the current status of translating optogenetic technology to primates, as well as how findings in rodents may help inform treatment of patients suffering from $O C D$ and related disorders. 


\section{Introduction}

Maladaptive repetitive actions and thoughts are key features of many neuropsychiatric illnesses, including autism [1], substance abuse disorders [2], Tourette's syndrome [3], and eating disorders [4]. The burden of these symptoms is perhaps most clearly highlighted in obsessive compulsive disorder (OCD), a chronic and severe mental illness affecting 2-3\% of people worldwide and identified as a leading cause of illness-related disability by the World Health Organization [5]. Recently separated from anxiety disorders in the DSM-5 based on proposed differences in neural substrates, subtypes of OCD are unified by the commonality of repetitive behaviors and/or thoughts that severely impair functioning. Several theories exist that may explain the pathophysiologic processes leading to compulsive behavior. Importantly, these proposed models posit dysfunction in distinct brain regions that comprise cortico-striato-thalamocortical (CSTC) loops, which are thought to be responsible for controlling action selection and behavioral inhibition [6]. Specific cortical and striatal regions within each loop have been linked to the development of either goal-directed or habitual behavior. In rodent studies, compulsive behavior has been linked to a progressive shift from recruitment of goal-directed systems to habitrelated systems $[2,7 \bullet]$, which has led to the proposal of the habit hypothesis of OCD [8]. However, the habit theory may not account for a key facet of compulsions in OCD-the intense urge to act [9•], which may be indicative of a behavior that is still goal-directed. Similar to models within the framework of addiction [10•], we propose that compulsive behaviors observed in OCD contain a substantial motivational and goal-directed component.

Here, we will summarize evidence from both human and preclinical animal studies linking dysfunctional CSTC activity to repetitive behaviors, and discuss potential implications of these findings for treatment. We will also highlight recent studies that investigate whether directly altering activity in discrete CSTC loops can change action strategies and affect compulsive behaviors. Based on convergent findings from animal and human OCD studies, we propose that compulsive behaviors may be induced either through increased habit system activity or enhanced recruitment of goal-directed systems.

\section{Neuroimaging studies highlight abnormalities in CSTC loops in} OCD

While the pathogenesis of OCD is still unknown, structural and functional neuroimaging studies have identified alterations in CSTC regions that are major players in the development of both goal-directed behaviors and habits in OCD patients compared to unaffected controls. Three general CSTC loops are believed to be important for controlling different neurocognitive functions, and are comprised of different interconnected brain regions [11]. These include the sensorimotor loop, which controls motor and response inhibition via lateral orbitofrontal cortex (IOFC) projections to the putamen; the cognitive loop, which regulates working memory via connections between dorsolateral prefrontal cortex (dlPFC) and the caudate nucleus; and the affective loop, which directs reward processing through interactions between the anterior cingulate cortex (ACC), the medial OFC (mOFC), and the ventral striatum [12, 13]. For each loop, information from the striatum is passed through the basal ganglia and ultimately conveyed back to the cortex via thalamic relays [11, 12]. Multiple lines of evidence suggest dysfunction in many of these regions-most of which contribute to producing goal-directed and habitual behavior-in OCD.

Functional magnetic resonance imaging (fMRI) studies highlight a role for both frontocortical structures (such as the OFC and ACC) and the striatum in OCD. Resting state studies suggest that, compared to healthy controls, OCD 
subjects have increased activity within both OFC and ACC [14, 15], as well as the caudate (dorsal striatum) [16]. OFC and caudate hyperactivity and ACC activity are further accentuated during symptom provocation, suggesting a potential casual role for these nuclei in the generation or expression of OCD symptoms $[17,18]$. Successful treatment of symptoms correlates with reduced activity in both caudate and OFC, regions associated with goal-directed action strategies [6], as measured by positron emission tomography and magnetic resonance spectroscopy [19-21]. In addition to elevated resting state activity, abnormal functional connectivity between these regions has also been observed. In particular, some (but not all, see [13]) data suggest increased functional connectivity between the ventral striatum/nucleus accumbens and medial OFC (mOFC) [22-24], a finding that is predictive of illness severity [25]. These functional imaging studies provide evidence that activity in regions involved in the development of both goal-directed and habitual behaviors is abnormal in OCD.

Though structural imaging studies have also suggested alterations in the caudate in both adults $[26,27]$ and children [28] with OCD, the directionality of change has not been consistent. In addition, increases in ventral striatal gray matter are observed in OCD subjects compared to controls and subjects with other anxiety disorders, implicating the ventral striatum in the formation of repetitive behavior rather than generalized anxiety [29]. Structural differences have also been detected in frontocortical regions. Specifically, decreased ACC volume has been reported in OCD patients $[29,30]$, and alterations in OFC gray matter volume in adult OCD have been observed [30-32] (though it is unclear if these changes are compensatory [33]). In addition to changes in gray matter density, abnormalities in white matter integrity have been identified using diffusion tensor imaging in corpus callosum, anterior limb of the internal capsule, and cingulum bundle. As with the aforementioned neurochemical and fMRI findings, decreases in white matter integrity within the left striatum of OCD patients can be reversed with chronic pharmacotherapy [34], suggesting that these changes may be central to the pathophysiology of the disorder. Although less consistent than functional imaging studies, structural MRI studies therefore identify alterations in OCD subjects within many of the same brain regions which are responsible for the development of goal-directed and habitual behavior.

Further evidence of CSTC dysfunction in OCD comes from invasive neurosurgical techniques used in treatment refractory OCD patients. It is estimated that $10-20 \%$ of patients with OCD do not respond to traditional pharmacological and psychological therapy [35-38]. Some of these individuals may benefit from treatments that directly target dysfunctional circuitry, including deep brain stimulation (DBS). DBS uses constant high-frequency electrical stimulation in targeted brain regions, and although the mechanism of action is unknown, some theories suggest that it is efficacious due to normalization of hyperactivity [19]. Currently, one of the most frequently studied DBS targets with substantial efficacy for OCD treatment is the ventral capsule/ventral striatum (VC/VS), with $25 \%$ of patients achieving remission after a year of stimulation [37-39]. VC/VS DBS normalized intraregion hyperactivity and reduced the increased functional activity observed between striatum and frontal cortex [40,41]. Again, this highlights the possible role of dysfunctional goaldirected behavior in OCD, since the VS is implicated in the prediction and 
evaluation of reward value, a necessary component of successful goal-directed action [2]. Together, these data highlight the involvement of regions that regulate habitual and goal-directed behavior in the pathophysiology of OCD, and provide hypotheses that can be tested utilizing new circuit-based interventions in animal models.

\section{Disruptions in habitual and goal-directed behavior in OCD}

Several recent studies in OCD patients have supported the hypothesis that compulsions stem from abnormal goal-directed and habitual striatal-mediated action strategies. First, in an instrumental conditioning paradigm, both OCD patients and controls learned to correctly discriminate between two visual stimuli at the same rate [42]. However, following devaluation of one of the two stimuli, OCD patients continued to respond to the devalued reward in a habitual fashion, reminiscent of rodents with dorsomedial striatum (DMS) lesions [43] (see "Circuits mediating goal-directed and habitual behavior in animal models"). Similarly, in an aversive conditioning paradigm, where correct discrimination of two visual stimuli leads to avoidance of a shock, no learning differences were detected $[44 \bullet \bullet]$. However, following overtraining and devaluation, OCD patients continued to avoid the devalued stimulus, despite the fact that they were consciously aware that it no longer predicted the shock. Using a modified version of the same task, researchers recently linked this increased habit formation back to CSTC circuits, finding increased activation of the caudate (DMS) in patients whose behavior had become habitual, and increased activation of the mOFC in all OCD subjects $[45 \bullet \bullet$. Together, these data suggest a shift in balance from goal-directed to habitual action selection strategy in OCD. However, it is worth noting that the degree of shock avoidance did not correlate with compulsion symptom severity (as measured by the YBOCS compulsion subscale), suggesting the bias towards a habitual strategy may not be relevant to OCD symptomatology. Further, OCD patients reported an increased urge to respond despite deval-

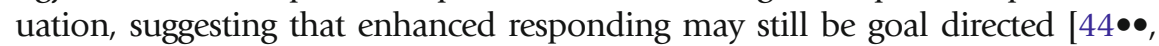
$45 \bullet \bullet$. These findings are broadly consistent with one current theory of drug addiction proposed by Sjoerds and colleagues [10•], in which compulsive drug seeking can be conceptualized as a maladaptive motivational habit with a substantial goal-directed component. Taken together with the identification of hyperactivity in regions linked to goal-directed behavior (the mOFC and caudate) by Gillan et al. [45••], it is possible that compulsivity in OCD may arise, at least for avoidance habits, from aberrant goal-directed recruitment.

Several other lines of evidence support a potential enhancement of the goaldirected action system in OCD. In experiments in which implicit (habitual) learning is examined, enhanced utilization of regions of the goal-directed system, including the OFC, is observed in OCD patients compared to controls $[46,47]$. When explicit learning or processing is introduced during the implicit task (thereby activating goal-directed circuitry), the performance of OCD patients compared to controls is impaired [48, 49]. A parsimonious explanation for these seemingly contradictory behavioral results, in which some data suggest an over-reliance on habit systems at the expense of goal-directed systems [42], while others indicate a strengthening of goal-directed actions in OCD [48, 49], has recently been summarized by Gruner and colleagues [50 $]$. However, the 
exact circuit level mechanisms regulating the inappropriate recruitment of either behavioral strategy are still not clear. Until recently, correlations between system-level disruptions in cortico-striatal-thalamo-cortical (CSTC) circuits and relevant behavioral abnormalities in OCD have provided the best available insight into the underlying neurobiology of OCD, since manipulation of specific neural circuits is still not possible in humans. Researchers have therefore now turned to animal models to address questions of causality.

\section{Circuits mediating goal-directed and habitual behavior in animal models}

To dissect the circuit mechanisms underlying the recruitment of goal-directed versus habitual behaviors, elegant studies have been performed in rodent models. This work has been essential to our understanding of drug addiction, which has been conceptualized as a disorder in which there is a gradual shift from recruitment of goal-directed systems to habit systems [2]. According to classic theory, the formation of habits arises when action-outcome driven (A-O) behaviors lead to learned stimulus-response (S-R) associations [51, 52]. Habitual behavior in normal animals (S-R driven) is typically induced by overtraining an animal during an operant task (e.g., pressing a lever for a reward). When the reward is then subsequently devalued through aversive conditioning, overtrained animals continue to respond despite the change in outcome, while normally trained animals respond less for the devalued outcome, indicative that the behavior remains goal-directed and flexible [53]. The neural basis for this change in strategy appears to lie in a shift from associative CSTC circuits to sensorimotor circuits, in analogous structures to those that are dysfunctional in OCD. Specifically, habitual responding can be induced via lesion of dorsomedial striatum (DMS: caudate in primates) [43, 54]. In contrast, disruption of the dorsolateral striatum (DLS: putamen in primates) promotes goal-directed behavior, even after overtraining and outcome devaluation $[54,55]$. Furthermore, substantial plasticity in these regions has been observed via in vivo recordings during habit formation. For example, during learning of a maze task, DLS activity rapidly shifts to a task-bracketing pattern during training, in which activity is high during both the beginning and end of a maze run [56]. This "chunking" pattern is believed to allow for more rapid and efficient neural processing during habitual behavior [57]. By contrast, activity in DMS is initially high at decision points during training and gradually decreases throughout overtraining, consistent with its role in action-outcome learning [56].

Less is known about upstream mediators that may regulate the shift between goal-directed and habitual action strategies. Lesion and inactivation studies suggest that two adjacent frontal cortical regions, the prelimbic (PL) and infralimbic (IL) cortices, may be important for regulating the switch between behavioral strategies. When activity of IL (akin to primate BA25) is inhibited, an animal's behavior becomes goal directed ([58]: inactivation, [59]: lesion). In contrast, when activity of PL (akin to primate BA32, part of anterior cingulate cortex) is disrupted, an animal's behavior becomes less goal directed [59, 60]. In addition to these regions, lesions of the lateral OFC in the monkey disrupt the ability to form action-outcome associations [61], indicating that OFC is also crucial for goal-directed decision making. 


\section{Development of optogenetics for investigation of complex behavior}

Although animal models have provided great insight into the brain regions mediating goal-directed versus habitual behavior, until recently, they were limited by the inability to manipulate specific cell types in particular neural circuits. This limitation has recently been surmounted by the development of optogenetics, a technique that leverages light-sensitive ion channels, known as opsins, for the precise activation and inhibition of neural circuits of interest. Although opsins were first isolated from microbial organisms approximately 40 years ago $[62,63]$, their utility for manipulating cellular activity has only recently been harnessed [64]. In the first wave of studies, two major opsins were used to bidirectionally alter cellular activity: (1) channelrhodopsin (ChR2), a cation channel that depolarizes cells in response to blue light [64, 65], and halorhodopsin (NpHR), a chloride pump that hyperpolarizes cells in response to yellow light $[66,67]$. As first reported in the seminal paper from Boyden and colleagues in 2005 [64], the expression of these light-sensitive ion channels in mammalian neurons combined with local light delivery $[68,69]$ allows for control of membrane excitability with millisecond temporal precision; this is significantly faster and more precise than traditional pharmacological methods. Significant efforts have since focused on expansion of the opsin family to improve kinetics, enhance photocurrents, limit toxicity, and broaden the range of usable light wavelengths. This has led to the creation of many valuable reagents, such as C1V1 [70] and ReaChR [71], which are red-shifted excitatory opsins; archaerhodopsin (ArchT), an outward driven proton pump that more efficiently hyperpolarizes cell membranes and therefore yields larger photocurrents compared to NpHR [72]; and more potent forms of ChR2, such as ChETA with improved kinetics [73], ChR2-TC that allows for lower intensity light stimulation [74], and step function opsins (SFO) with which single light pulses can stably alter membrane potential [75].

In addition to unrivaled temporal resolution, optogenetics provides unique advantages over traditional pharmacological and electrical stimulation, including spatial resolution and targeting to genetically distinct cell populations via stereotactic injection of viral vectors under the control of cell type-specific promoters [76]. Additional specificity can be gained using combinatorial genetic approaches that include virally-expressing opsins only in cells with genetically encoded crerecombinase, a strategy which has now been successfully used many times to manipulate small populations of cells during behavioral tasks [77-79]. Furthermore, optogenetic methods allow for the dissection of microcircuits on a scale not previously possible. Following injection of an opsin-encoding virus (either promoter or cre-dependent) into a region of interest, infected cells will express the channel or pump throughout the cell membrane, including the axon terminals. Implanting a light source into the terminal region allows for precise manipulation of very specific projections within a microcircuit rather than pan-activation of cell bodies that project to multiple target sites (see Fig. 1 and Tye and Deisseroth [80] for detailed description). As we will highlight later in this review, this technique can be used to tease apart the contributions of subregion-specific projections to complex behaviors. 


\section{Optogenetic manipulation of habit and goal-directed circuits}

Over the past 5 years, optogenetics has been utilized to more precisely define the circuits that control goal-directed versus habitual behavior according to traditional lesion and inactivation studies, as well as to determine how activity of these circuits may contribute to OCD-relevant behavior. In 2013, Gremel and Costa established a behavioral paradigm to better understand the mechanisms underlying the shift from goal-directed to habitual actions. To test whether separate neural ensembles within CSTC circuits control goal-directed versus habitual behavior, or whether two types of action strategies are encoded by identical ensembles, they constructed a novel behavioral task in which animals performed an identical action (a lever press) using either a goal-directed (random ratio) or habitual (random interval) strategy. As predicted based on prior studies, lesions to DMS resulted in habitual behavior in both conditions, while lesions to DLS led to goal-directed behavior. Lesioning IOFC had the same effect as a DMS lesion-i.e., producing habitual responding. These data suggest that the IOFC and DMS are critical for encoding changes in outcome value. In order to test the necessity and sufficiency of lOFC activation in encoding outcome value, the authors next conducted chemogenetic loss of function [81, 82] and optogenetic gain of function studies. After injection of a virus encoding the inhibitory designer receptor exclusively activated by designer drug (DREADD) $\left(\mathrm{hM} 4 \mathrm{D}_{\mathrm{i}}\right)$ into the lOFC and subsequent inhibition via administration of its ligand clozapine-N-oxide (CNO), mice behaved in a habitual manner, similarly to performance following an OFC lesion. Based on these findings, the authors hypothesized that activation of OFC projection neurons would enhance goal-directed behavior. Bilateral activation of IOFC cell bodies via optical stimulation had no effect when the reward was still valued in the random ratio condition. Instead, it drastically increased lever pressing when the reward was devalued, resulting in levels of pressing similar to non-devalued rats, which the authors interpreted as an enhancement of goal-directed behavior. In total, these data support previous studies suggesting that goal-directed behavior is controlled by the DMS, while habitual behavior is subserved by the DLS $[43,83]$. Furthermore, they confirm the role of the OFC in encoding value and goal-directed behavior $[6,84]$.

Optogenetics has also been used to investigate the contribution of affective/ prefrontal circuits to the shift from goal-directed to habitual behavior. These experiments focused on manipulating the infralimbic PFC (IL), which directly projects to the DMS $[59,60]$ and may indirectly project to the DLS via the ventral striatum or the amygdala [85]. A dual pattern of connections to the sensorimotor network (DLS; promoting habitual behavior) and to regions that promote goal-directed behavior (DMS) suggests that the IL may exert executive control over habit formation. To assess the real-time impact of IL activity on habit formation, Smith and colleagues [ $88 \bullet \bullet$ ] selectively expressed halorhodopsin (NpHR3) bilaterally in IL pyramidal cells. Based on tones, rats then learned to predict which arm of a T-maze contained one of two food rewards. After overtraining, rats received home-cage devaluation of one of the rewards through conditioned taste aversion. After devaluation, control rats continued to run to the devalued arm, suggesting this behavior had become habitual. In contrast, rats that received optical inhibition of the IL immediately after devaluation generally ran to the non-devalued arm, suggesting that IL inhibition blocked habit expression. Consistent with literature suggesting that 


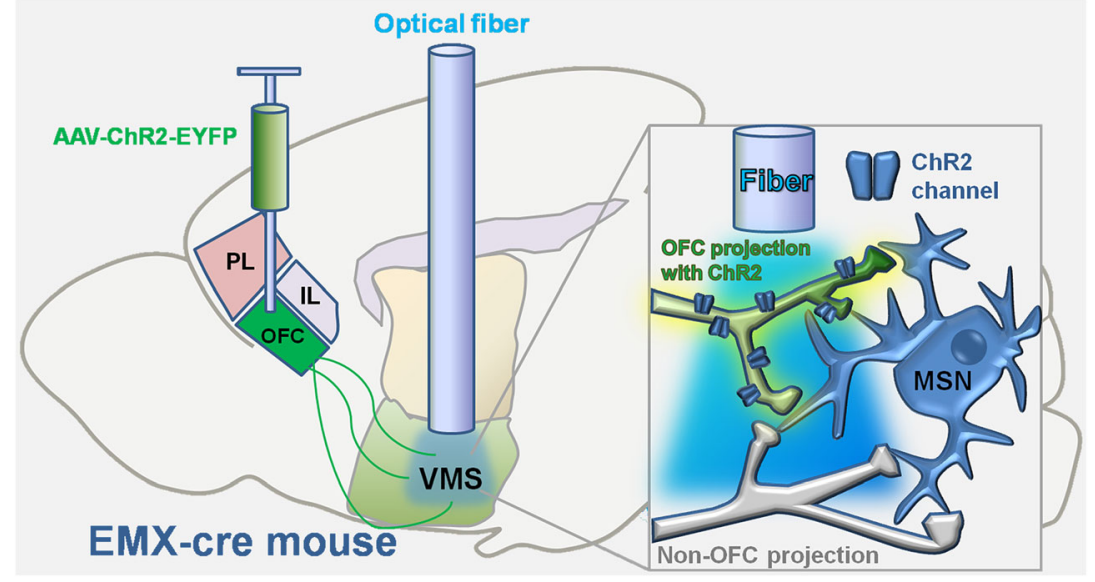

Fig. 1. Projection-specific expression and activation of excitatory opsin (ChR2) to dissect OCD-related circuitry. Presynaptic neurons within the OFC of EMX-cre mice are transduced with ChR2-EYFP. An optical fiber is implanted into the target region (VMS) and blue light (473 nm wavelength) is delivered to excite infected projection neurons. Inset Blue light stimulation selectively activates OFC projection neurons infected with ChR2, resulting in depolarization of post-synaptic medium spiny neurons (MSNs) selectively connected to OFC. Uninfected projection neurons from the OFC or from other regions are unaffected by optical stimulation.

once a habit is broken it is often replaced with another habit, all rats that received devaluation began to run consistently to the non-devalued reward in response to the devalued tone. These responses increased over time in both groups, suggestive of new habit formation. Strikingly, when IL optical inhibition was performed 2 weeks post-devaluation, the rats immediately ran back to the devalued arm. This suggests that, as with initial habit formation, the IL was responsible for the establishment of the new habit. These data support the idea that inhibition of the IL restores previously learned action strategies once a new habit is ingrained [59]. Consistent with this interpretation, while in vivo activity in the DLS exhibited characteristic task bracketing during the middle of acquisition of this T-maze task $[56,86]$, activity in the IL only became task bracketed

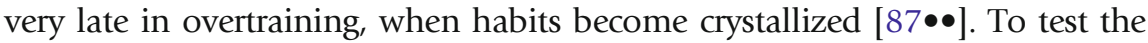
significance of IL task bracketing during overtraining, rats received bilateral injection of NpHR3 in the IL and underwent T-maze training in an independent

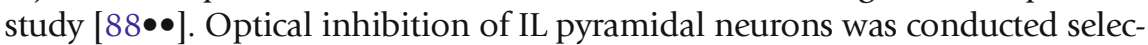
tively during overtraining, when task-bracketing activity appeared during recording. As predicted, habit formation was blocked in rats with IL perturbation during overtraining $[87 \bullet \bullet]$. Together, these data suggest that IL activity has a role in both the formation and the expression of habits. Recent computational work in humans corroborates these findings, implicating the inferior lateral PFC (ilPFC) and frontopolar cortex (FPC) as potential arbitrators of the switch from goal-directed to habitual behavior through connections with the putamen [89].

\section{Optogenetic manipulation of circuits underlying compulsive} behavior

Two recent optogenetic studies have begun to explore the relationship of the hyperactivity of CSTC pathways observed in human OCD to the development and resolution of perseverative behavior. An experiment conducted by Burguiere and colleagues [92] used a transgenic mouse model of OCD-like behavior based on deletion of Sapap3 (SAP90/PSD95-associated protein 3), which is found in the post-synaptic density of glutamatergic synapses and 
interacts with PSD95 and other scaffolding proteins [90]. Genetic variants of SAPAP3 have been linked to OCD and grooming disorders, such as trichotillomania and skin picking ([91, 92] correspondingly), and Sapap3 knockout (KO) mice exhibit OCD-related behaviors including compulsive over-grooming and increased anxiety [93]. In this study, an aversive conditioning paradigm was used to train mice to associate a tone with delivery of a drop of water on their head. As training progressed, both wild-type and SAPAP3 KOs successfully learned that the tone predicted the water drop and groomed in response to the tone. However, wild-type mice began to inhibit this response late in training, only grooming after the water drop was presented. In contrast, Sapap3 KOs were unable to inhibit the response to the tone, and instead, continued to groom after tone presentation

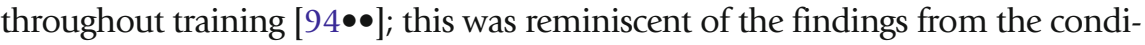
tioning experiments in OCD patients described above. The authors then characterized the neural underpinnings of this impaired behavioral inhibition, finding that the deficit was correlated with elevated striatal activity in Sapap3 KOs. Supporting the hypothesis that this increased activity of striatal medium spiny neurons (MSNs) resulted from a loss of striatal inhibitory interneurons, they observed that Sapap3 KOs had fewer parvalbumin-positive (PV+) interneurons in the centromedial striatum (CMS). In an attempt to correct for this deficit, they then performed selective ChR2-mediated optogenetic stimulation of projections from IOFC to CMS. This intervention both reversed the deficit in behavioral inhibition and the increased striatal activity, presumably via enhancing the responsiveness of the remaining PV-positive interneurons and restoring normal feed-forward inhibition. These experiments provided support for the idea that IOFC regulates the inhibition of conditioned responses, and linked hyperactivity in striatum to compulsive behavior.

In a complementary set of studies, our lab used optogenetics to test whether inducing hyperactivity in CSTC circuits in wild-type mice directly leads to abnormal repetitive behaviors. Focusing on the subregions that have been implicated in goal-directed behavior and anxiety, we selectively expressed ChR2 in excitatory projections from mOFC to ventromedial striatum (VMS). Although acute optogenetic stimulation did not lead to OCD-related behavioral changes, brief but repeated stimulation of mOFC-VMS projections led to a significant increase in perseverative grooming over the course of 5-6 days [95••]. Strikingly, this increase in grooming was persistent for up to 2 weeks after cessation of the optogenetic stimulation. Together, these findings suggested the development of pathologic plasticity in mOFC-VMS connections. In support of this concept, using in vivo recording we demonstrated a progressive increase in evoked firing rates of VMS neurons over 5 days of stimulation. Lending support to the idea that these plastic changes may have relevance to pathologic processes involved in $\mathrm{OCD}$, both the increased grooming behavior and the increase in evoked firing were normalized by chronic SSRI administration. Our data suggest that brief but repeated hyperstimulation of regions implicated in both goal-directed behavior (mOFC and medial striatum) and limbic/anxiety circuits (VMS) can lead to perseverative behavior that may be relevant to OCD. These data therefore support the hypothesis that hyperactivity in goal-directed circuits can lead to the development of abnormal repetitive behaviors. Future studies will seek to synthesize these two sets of findings, which together suggest there may be two paths to producing perseverative behavior-i.e., through dysfunction of either habit systems or goal-directed/limbic systems. 


\section{Summary and conclusions}

Over the past 5 years, optogenetic experiments have led to rapid progress in dissecting the functional relationship between CSTC circuits and the development of OCD-relevant behaviors in animal models. Chief among these findings are the importance of different prefrontal and striatal subregions in the control of goal-directed versus habitual behavior, and direct tests of the hypotheses that hyperactivity in OFC and striatal circuitry is involved in OCD-relevant behavioral phenotypes in normal and pathological animals $[94 \bullet \bullet, 95 \bullet \bullet, 96 \bullet \bullet]$. These findings provide a causal link to some of the most well-replicated findings in human OCD imaging studies, demonstrating OFC hyperactivity and altered functional connectivity between OFC and the medial striatum $[25,29]$. Interestingly, involvement of the mOFC suggests that an over-reliance on the goaldirected system (including the mOFC and medial striatum) may play a role in the development of compulsive behavior [50 ]. These data are particularly interesting in light of recent neuroimaging findings in which increased avoidance habit formation in OCD patients was associated with hyperactivation of mOFC and medial striatum, classically thought of as regions promoting goaldirected behavior $[43,45 \bullet \bullet, 60]$. Future work will determine how other regions outside of the CSTC involved in anxiety and reward, such as the amygdala, ventral hippocampus, and VTA, may be involved in mediating compulsive behavior.

\section{Future directions and prospective implications for treatment of $O C D$ and related disorders}

Optogenetics has allowed for unparalleled precise control in animal model systems over circuits implicated in OCD and known to be involved in goaldirected and habitual behavior. Future research will focus on elucidating the molecular mechanisms underlying not only the onset of compulsive and perseverative behaviors, but also their persistence. The striking findings of

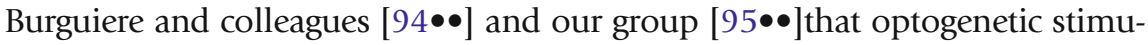
lation of disease-relevant neural circuits can alter perseverative behaviors raises the attractive possibility of optogenetics as a potential therapeutic tool for OCD and related disorders. However, substantial hurdles exist before this technology can be translated to humans, including synthesizing viruses that are both safe for humans and can effectively target specific cell types. In addition, experiments using optogenetics in primates to date have had substantial difficulty in generating the striking behavioral effects that are commonly observed in rodent studies $[72,97]$; the reasons for this are unclear, but must be determined before progress can be made towards using the technology in people. Finally, further progress still needs to be made towards development of wireless systems for effective stimulation, though this goal is clearly within reach [97]. More practically, optogenetics will continue to be utilized as a valuable tool for dissection of circuits known to be dysfunctional in human disease. Indeed, the potential combination of optogenetics and fMRI [98] or in vivo calcium imaging [99] provides important avenues for enhancing our understanding of how 
hyperactivity of a single pathway (e.g., OFC to VMS) can impact brain-wide networks. Critically, the information gained from these targeted approaches will inform therapeutics either through the refinement of current technologies, such as DBS and transcranial magnetic stimulation (TMS), or through the development of new technologies, such as closed loop systems that would allow for real-time adjustment of stimulation parameters based on abnormal neural activity patterns [100].

\section{Compliance with Ethics Guidelines}

\section{Conflict of Interest}

Sean C. Piantadosi reports grants from NIMH, during the conduct of the study. Susanne E. Ahmari reports grants from NIMH during the conduct of the study.

\section{Human and Animal Rights and Informed Consent}

All experimental procedures in referenced work by S.E.A. were approved by the University of Pittsburgh and Columbia University Institutional Animal Care and Use Committees, and were conducted in accordance with the National Institutes of Health (NIH) Guide for the Care and Use of Laboratory Animals. This article does not contain any studies with human or animal subjects performed by any of the authors.

\section{References and Recommended Reading}

Papers of particular interest, published recently, have been highlighted as:

- Of importance

$\bullet \quad$ Of major importance

1. McDougle CJ, Kresch LE, Goodman WK, Naylor ST, Volkmar FR, Cohen DJ, et al. A case-controlled study of repetitive thoughts and behavior in adults with autistic disorder and obsessive-compulsive disorder. Am J Psychiatry. 1995;152:772-7.

2. Everitt BJ, Robbins TW. Neural systems of reinforcement for drug addiction: from actions to habits to compulsion. Nat Neurosci. 2005;8:1481-9.

3. Diagnostic and statistical manual of mental disorders, (DSM-5 ${ }^{\circledR}$ ): American Psychiatric Pub; 2013.

4. Smith DG, Robbins TW. The neurobiological underpinnings of obesity and binge eating: a rationale for adopting the food addiction model. Biol Psychiatry. 2013;73:804-10.

5. Kessler RC, Berglund P, Demler O, Jin R, Merikangas $\mathrm{KR}$, Walters EE. Lifetime prevalence and age-of-onset distributions of DSM-IV disorders in the National Comorbidity Survey Replication. Arch Gen Psychiatry. 2005;62:593-602.

6. Valentin VV, Dickinson A, O'Doherty JP. Determining the neural substrates of goal-directed learning in the human brain. J Neurosci Off J Soc Neurosci. 2007;27:4019-26.
7. $\quad$ Everitt BJ, Robbins TW. From the ventral to the dorsal striatum: devolving views of their roles in drug addiction. Neurosci Biobehav Rev. 2013;37:1946-54.

This review summarizes what is known regarding the neural substrates underlying the transition from goal-directed to habitual behavior in models of compulsive drug seeking.

8. Graybiel AM, Rauch SL. Toward a neurobiology of obsessive-compulsive disorder. Neuron. 2000;28:3437.

9.• Denys D. Compulsivity and free will. CNS Spectr. 2014;19:8-9.

This editorial discusses the notion of compulsivity as an internally generated goal-directed drive to act.

10.• Sjoerds Z, Luigjes J, van den Brink W, Denys D, Yücel $M$. The role of habits and motivation in human drug addiction: a reflection. Front Psychiatry. 2014;5:8.

This paper differentiates motor habits from "motivational" habits seen in compulsive drug seeking, which contains a substantial goal-directed component.

11. Milad MR, Rauch SL. Obsessive-compulsive disorder: beyond segregated cortico-striatal pathways. Trends Cogn Sci. 2012;16:43-51. 
12. Di Martino A, Scheres A, Margulies DS, Kelly AM, Uddin LQ, Shehzad Z, et al. Functional connectivity of human striatum: a resting state FMRI study. Cereb Cortex. 2008;18:2735-47.

13. Posner J, Marsh R, Maia TV, Peterson BS, Gruber A, Simpson HB. Reduced functional connectivity within the limbic cortico-striato-thalamo-cortical loop in unmedicated adults with obsessive-compulsive disorder. Hum Brain Mapp. 2014;35:2852-60.

14. Baxter LR, Phelps ME, Mazziotta JC, Guze BH, Schwartz $\mathrm{JM}$, Selin CE. Local cerebral glucose metabolic rates in obsessive-compulsive disorder. A comparison with rates in unipolar depression and in normal controls. Arch Gen Psychiatry. 1987;44:211-8.

15. Swedo SE, Schapiro MB, Grady CL, Cheslow DL, Leonard HL, Kumar A, et al. Cerebral glucose metabolism in childhood-onset obsessive-compulsive disorder. Arch Gen Psychiatry. 1989;46:518-23.

16. Nordahl TE, Benkelfat C, Semple WE, Gross M, King AC, Cohen RM. Cerebral glucose metabolic rates in obsessive compulsive disorder.

Neuropsychopharmacol Off Publ Am Coll

Neuropsychopharmacol. 1989;2:23-8.

17. Rauch SL, Jenike MA, Alpert NM, Baer L, Breiter HC, Savage CR, et al. Regional cerebral blood flow measured during symptom provocation in obsessivecompulsive disorder using oxygen 15-labeled carbon dioxide and positron emission tomography. Arch Gen Psychiatry. 1994;51:62-70.

18. Adler CM, McDonough-Ryan P, Sax KW, Holland SK, Arndt S, Strakowski SM. fMRI of neuronal activation with symptom provocation in unmedicated patients with obsessive compulsive disorder. J Psychiatr Res. 2000;34:317-24.

19. Abelson JL, Curtis GC, Sagher O, Albucher RC, Harrigan M, Taylor SF, et al. Deep brain stimulation for refractory obsessive-compulsive disorder. Biol Psychiatry. 2005;57:510-6.

20. Rauch SL, Shin LM, Dougherty DD, Alpert NM, Fischman AJ, Jenike MA. Predictors of fluvoxamine response in contamination-related obsessive compulsive disorder: a PET symptom provocation study. Neuropsychopharmacology. 2002;27:782-91.

21. Rosenberg DR, MacMaster FP, Keshavan MS, Fitzgerald KD, Stewart CM, Moore GJ. Decrease in caudate glutamatergic concentrations in pediatric obsessivecompulsive disorder patients taking paroxetine. J Am Acad Child Adolesc Psychiatry. 2000;39:1096-103.

22. Harrison BJ, Soriano-Mas C, Pujol J, Ortiz H, LópezSolà M, Hernández-Ribas R, et al. Altered corticostriatal functional connectivity in obsessive-compulsive disorder. Arch Gen Psychiatry. 2009;66:1189-200.

23. Fitzgerald KD, Welsh RC, Stern ER, Angstadt M, Hanna GL, Abelson JL, et al. Developmental alterations of frontal-striatal-thalamic connectivity in obsessivecompulsive disorder. J Am Acad Child Adolesc Psychiatry. 2011;50:938-948000.

24. Hou J, Wu W, Lin Y, Wang J, Zhou D, Guo J, et al. Localization of cerebral functional deficits in patients with obsessive-compulsive disorder: a resting-state fMRI study. J Affect Disord. 2012;138:313-21.

25. Harrison BJ, Pujol J, Cardoner N, Deus J, Alonso P, López-Solà $\mathrm{M}$, et al. Brain corticostriatal systems and the major clinical symptom dimensions of obsessivecompulsive disorder. Biol Psychiatry. 2013;73:321-8.

26. Robinson D, Wu H, Munne RA, Ashtari M, Alvir JM, Lerner $\mathrm{G}$, et al. Reduced caudate nucleus volume in obsessive-compulsive disorder. Arch Gen Psychiatry. 1995;52:393-8.

27. Scarone S, Colombo C, Livian S, Abbruzzese M, Ronchi $\mathrm{P}$, Locatelli $\mathrm{M}$, et al. Increased right caudate nucleus size in obsessive-compulsive disorder: detection with magnetic resonance imaging. Psychiatry Res. 1992;45:11521.

28. Rosenberg DR, Keshavan MS, O'Hearn KM, Dick EL, Bagwell WW, Seymour AB, et al. Frontostriatal measurement in treatment-naive children with obsessivecompulsive disorder. Arch Gen Psychiatry. 1997;54:824-30.

29. Menzies L, Chamberlain SR, Laird AR, Thelen SM, Sahakian BJ, Bullmore ET. Integrating evidence from neuroimaging and neuropsychological studies of obsessive-compulsive disorder: the orbitofrontostriatal model revisited. Neurosci Biobehav Rev. 2008;32:525-49.

30. Radua J, van den Heuvel OA, Surguladze S, MataixCols D. Meta-analytical comparison of voxel-based morphometry studies in obsessive-compulsive disorder vs other anxiety disorders. Arch Gen Psychiatry. 2010;67:701-11.

31. Rotge J-YY, Guehl D, Dilharreguy B, Tignol J, Bioulac B, Allard $\mathrm{M}$, et al. Meta-analysis of brain volume changes in obsessive-compulsive disorder. Biol Psychiatry. 2009;65:75-83.

32. Atmaca M, Yildirim H, Ozdemir H, Tezcan E, Poyraz AK. Volumetric MRI study of key brain regions implicated in obsessive-compulsive disorder. Prog NeuroPsychopharmacol Biol Psychiatry. 2007;31:46-52.

33. Radua J, Mataix-Cols D. Voxel-wise meta-analysis of grey matter changes in obsessive-compulsive disorder. Br J Psychiatry J Mental Sci. 2009;195:393-402.

34. Fan Q, Yan X, Wang J, Chen Y, Wang X, Li C, et al. Abnormalities of white matter microstructure in unmedicated obsessive-compulsive disorder and changes after medication. PLoS One. 2012;7:e35889.

35. Husted DS, Shapira NA. A review of the treatment for refractory obsessive-compulsive disorder: from medicine to deep brain stimulation. CNS Spectr. 2004;9:833-47.

36. Rodman AM, Milad MR, Deckersbach T, Im J, Chou T, Dougherty DD. Neuroimaging contributions to novel surgical treatments for intractable obsessivecompulsive disorder. Expert Rev Neurother. 2012;12:219-27.

37. Greenberg BD, Gabriels LA, Malone DA, Rezai AR, Friehs GM, Okun MS, et al. Deep brain stimulation of the ventral internal capsule/ventral striatum for 
obsessive-compulsive disorder: worldwide experience. Mol Psychiatry. 2010;15:64-79.

38. Denys $\mathrm{D}$, Mantione $\mathrm{M}$, Figee $\mathrm{M}$, van den Munckhof $\mathrm{P}$, Koerselman F, Westenberg H, et al. Deep brain stimulation of the nucleus accumbens for treatmentrefractory obsessive-compulsive disorder. Arch Gen Psychiatry. 2010;67:1061-8.

39. Greenberg BD, Rauch SL, Haber SN. Invasive circuitrybased neurotherapeutics: stereotactic ablation and deep brain stimulation for OCD.

Neuropsychopharmacol Off Publ Am Coll Neuropsychopharmacol. 2010;35:317-36.

40. Figee $M$, Luigjes J, Smolders R, Valencia-Alfonso C-EE, van Wingen G, de Kwaasteniet B, et al. Deep brain stimulation restores frontostriatal network activity in obsessive-compulsive disorder. Nat Neurosci. 2013;16:386-7.

41. Figee M, Wielaard I, Mazaheri A, Denys D. Neurosurgical targets for compulsivity: what can we learn from acquired brain lesions? Neurosci Biobehav Rev. 2013;37:328-39.

42. Gillan CM, Papmeyer M, Morein-Zamir S, Sahakian BJ, Fineberg NA, Robbins TW, et al. Disruption in the balance between goal-directed behavior and habit learning in obsessive-compulsive disorder. Am J Psychiatry. 2011;168:718-26.

43. Yin HH, Knowlton BJ, Balleine BW. Blockade of NMDA receptors in the dorsomedial striatum prevents actionoutcome learning in instrumental conditioning. Eur J Neurosci. 2005;22:505-12.

44.• Gillan CM, Morein-Zamir S, Urcelay GP, Sule A, Voon $\mathrm{V}$, Apergis-Schoute AM, et al. Enhanced avoidance habits in obsessive-compulsive disorder. Biol Psychiatry. $2014 ; 75: 631-8$.

This paper describes the discovery that OCD patients have maladaptive avoidance habits.

45.• Gillan CM, Apergis-Schoute AM, Morein-Zamir S, Urcelay GP, Sule A, Fineberg NA, et al. Functional neuroimaging of avoidance habits in obsessive-compulsive disorder. Am J Psychiatry. 2015;172:284-93.

This paper describes neural correlates of mOFC and caudate hyperactivity in OCD patients that develop enhanced avoidance habits.

46. Rauch SL, Savage CR, Alpert NM, Dougherty D, Kendrick A, Curran T, et al. Probing striatal function in obsessive-compulsive disorder: a PET study of implicit sequence learning. J Neuropsychiatry Clin Neurosci. 1997;9:568-73.

47. Rauch SL, Wedig MM, Wright CI, Martis B, McMullin KG, Shin LM, et al. Functional magnetic resonance imaging study of regional brain activation during implicit sequence learning in obsessive-compulsive disorder. Biol Psychiatry. 2007;61:330-6.

48. Deckersbach T, Savage CR, Curran T, Bohne A, Wilhelm $\mathrm{S}$, Baer L, et al. A study of parallel implicit and explicit information processing in patients with obsessivecompulsive disorder. Am J Psychiatry. 2002;159:1780-2.

49. Joel D, Zohar O, Afek M, Hermesh H, Lerner L, Kuperman R, et al. Impaired procedural learning in obsessive-compulsive disorder and Parkinson's disease, but not in major depressive disorder. Behav Brain Res. 2005;157:253-63.

50. Gruner P, Anticevic A, Lee D, Pittenger C. Arbitration between action strategies in obsessive-compulsive disorder. Neuroscientist Rev J Neurobiol Neurol Psychiatry. 2015. doi:10.1177/1073858414568317.

This review comprehensively examines current evidence linking pathological action strategy (goal directed and habit) utilization in OCD.

51. Adams C. Variations in the sensitivity of instrumental responding to reinforcer devaluation. Q J Exp Psychol B. 2007;34:7798.

52. Balleine B, Dickinson A. Signalling and incentive processes in instrumental reinforcer devaluation. Q J Exp Psychol B Comp Physiol Psychol. 1992;45:285-301.

53. Yin HH, Knowlton BJ, Balleine BW. Inactivation of dorsolateral striatum enhances sensitivity to changes in the action-outcome contingency in instrumental conditioning. Behav Brain Res. 2006;166:189-96.

54. Yin HH, Knowlton BJ, Balleine BW. Lesions of dorsolateral striatum preserve outcome expectancy but disrupt habit formation in instrumental learning. Eur J Neurosci. 2004;19:181-9.

55. Yin HH, Knowlton BJ. The role of the basal ganglia in habit formation. Nat Rev Neurosci. 2006;7:464-76.

56. Thorn CA, Atallah H, Howe M, Graybiel AM. Differential dynamics of activity changes in dorsolateral and dorsomedial striatal loops during learning. Neuron. 2010;66:781-95.

57. Graybiel AM. Habits, rituals, and the evaluative brain. Annu Rev Neurosci. 2008;31:359-87.

58. Coutureau E, Killcross S. Inactivation of the infralimbic prefrontal cortex reinstates goal-directed responding in overtrained rats. Behav Brain Res. 2003;146:167-74.

59. Killcross S, Coutureau E. Coordination of actions and habits in the medial prefrontal cortex of rats. Cerebr Cortex (New York, NY: 1991). 2003;13:400-8.

60. Balleine BW, Dickinson A. Goal-directed instrumental action: contingency and incentive learning and their cortical substrates. Neuropharmacology. 1998;37:407-19.

61. Rudebeck PH, Murray EA. Dissociable effects of subtotal lesions within the macaque orbital prefrontal cortex on reward-guided behavior. J Neurosci Off J Soc Neurosci. 2011;31:10569-78.

62. Oesterhelt D, Stoeckenius W. Rhodopsin-like protein from the purple membrane of Halobacterium halobium. Nat New Biol. 1971;233:149-52.

63. Matsuno-Yagi A, Mukohata Y. Two possible roles of bacteriorhodopsin; a comparative study of strains of Halobacterium halobium differing in pigmentation. Biochem Biophys Res Commun. 1977;78:237-43.

64. Boyden ES, Zhang F, Bamberg E, Nagel G, Deisseroth K. Millisecond-timescale, genetically targeted optical control of neural activity. Nat Neurosci. 2005;8:1263-8.

65. Nagel G, Ollig D, Fuhrmann M, Kateriya S, Musti AM, Bamberg E, et al. Channelrhodopsin-1: a light-gated proton channel in green algae. Science (New York, NY). 2002;296:2395-8. 
66. Zhang F, Aravanis AM, Adamantidis A, de Lecea L, Deisseroth K. Circuit-breakers: optical technologies for probing neural signals and systems. Nat Rev Neurosci. 2007;8:577-81.

67. Zhang F, Wang L-PP, Brauner M, Liewald JF, Kay K, Watzke N, et al. Multimodal fast optical interrogation of neural circuitry. Nature. 2007;446:633-9.

68. Aravanis AM, Wang L-PP, Zhang F, Meltzer LA, Mogri $M Z$, Schneider MB, et al. An optical neural interface: in vivo control of rodent motor cortex with integrated fiberoptic and optogenetic technology. J Neural Eng. 2007;4:56.

69. Arenkiel BR, Peca J, Davison IG, Feliciano C, Deisseroth $\mathrm{K}$, Augustine GJ, et al. In vivo light-induced activation of neural circuitry in transgenic mice expressing channelrhodopsin-2. Neuron. 2007;54:205-18.

70. Erbguth K, Prigge M, Schneider F, Hegemann P, Gottschalk A. Bimodal activation of different neuron classes with the spectrally red-shifted channelrhodopsin chimera C1V1 in Caenorhabditis elegans. PLoS One. 2012;7:e46827.

71. Lin JY, Knutsen PM, Muller A, Kleinfeld D, Tsien RY. ReaChR: a red-shifted variant of channelrhodopsin enables deep transcranial optogenetic excitation. Nat Neurosci. 2013;16:1499-508.

72. Han X, Chow BY, Zhou H, Klapoetke NC, Chuong A, Rajimehr R, et al. A high-light sensitivity optical neural silencer: development and application to optogenetic control of non-human primate cortex. Front Syst Neurosci. 2011;5:18.

73. Gunaydin LA, Yizhar O, Berndt A, Sohal VS, Deisseroth K, Hegemann P. Ultrafast optogenetic control. Nat Neurosci. 2010;13:387-92.

74. Berndt A, Schoenenberger P, Mattis J, Tye KM, Deisseroth K, Hegemann P, et al. High-efficiency channelrhodopsins for fast neuronal stimulation at low light levels. Proc Natl Acad Sci U S A. 2011;108:7595-600.

75. Berndt A, Yizhar O, Gunaydin LA, Hegemann P, Deisseroth K. Bi-stable neural state switches. Nat Neurosci. 2009;12:229-34.

76. Mattis J, Tye KM, Ferenczi EA, Ramakrishnan C, O'Shea DJ, Prakash R, et al. Principles for applying optogenetic tools derived from direct comparative analysis of microbial opsins. Nat Methods. 2012;9:159-72.

77. Tsai H-CC, Zhang F, Adamantidis A, Stuber GD, Bonci A, de Lecea $\mathrm{L}$, et al. Phasic firing in dopaminergic neurons is sufficient for behavioral conditioning. Science (New York, NY). 2009;324:1080-4.

78. Witten IB, Lin S-CC, Brodsky M, Prakash R, Diester I, Anikeeva $\mathrm{P}$, et al. Cholinergic interneurons control local circuit activity and cocaine conditioning. Science (New York, NY). 2010;330:1677-81.

79. Fenno L, Yizhar O, Deisseroth K. The development and application of optogenetics. Annu Rev Neurosci. 2011;34:389-412.

80. Tye KM, Deisseroth K. Optogenetic investigation of neural circuits underlying brain disease in animal models. Nat Rev Neurosci. 2012;13:251-66.
81. Alexander GM, Rogan SC, Abbas AI, Armbruster BN, Pei Y, Allen JA, et al. Remote control of neuronal activity in transgenic mice expressing evolved G protein-coupled receptors. Neuron. 2009;63:27-39.

82. Pei Y, Dong S, Roth BL. Generation of designer receptors exclusively activated by designer drugs (DREADDs) using directed molecular evolution. Current protocols in neuroscience / editorial board, Jacqueline N. Crawley ... [et al.]. 2010;Chapter 4.

83. Dezfouli A, Balleine BW. Habits, action sequences and reinforcement learning. Eur J Neurosci. 2012;35:103651.

84. Damasio AR, Tranel D, Damasio H. Individuals with sociopathic behavior caused by frontal damage fail to respond autonomically to social stimuli. Behav Brain Res. 1990;41:81-94.

85. Hurley KM, Herbert H, Moga MM, Saper CB. Efferent projections of the infralimbic cortex of the rat. J Comp Neurol. 1991;308:249-76.

86. Graybiel AM. The basal ganglia and chunking of action repertoires. Neurobiol Learn Mem. 1998;70:119-36.

87.• Smith KS, Graybiel AM. A dual operator view of habitual behavior reflecting cortical and striatal dynamics. Neuron. 2013;79:361-74.

In this paper, the authors expand on previous findings and demonstrate differential action-bracketing spiking activity in the DLS and the infralimbic cortex during overtraining in rats, which was disrupted with cortical optogenetic inhibition.

88.•• Smith KS, Virkud A, Deisseroth K, Graybiel AM. Reversible online control of habitual behavior by optogenetic perturbation of medial prefrontal cortex. Proc Natl Acad Sci U S A. 2012;109:18932-7.

In this paper, the authors utilize optogenetic inhibition to characterize a key role of the infralimbic cortex in habit formation.

89. Lee SW, Shimojo S, O'Doherty JP. Neural computations underlying arbitration between model-based and model-free learning. Neuron. 2014;81:687-99.

90. Takeuchi M, Hata Y, Hirao K, Toyoda A, Irie M, Takai Y. SAPAPs. A family of PSD-95/SAP90-associated proteins localized at postsynaptic density. J Biol Chem. 1997;272:11943-51.

91. Bienvenu OJ, Wang Y, Shugart YY, Welch JM, Grados MA, Fyer AJ, et al. Sapap3 and pathological grooming in humans: results from the OCD collaborative genetics study. Am J Med Genet B Neuropsychiatr Genet Off Publ Int Soc Psychiatr Genet. 2009;150B:710-20.

92. Züchner S, Wendland JR, Ashley-Koch AE, Collins AL, Tran-Viet KN, Quinn K, et al. Multiple rare SAPAP3 missense variants in trichotillomania and OCD. Mol Psychiatry. 2009;14:6-9.

93. Welch JM, Lu J, Rodriguiz RM, Trotta NC, Peca J, Ding J$\mathrm{DD}$, et al. Cortico-striatal synaptic defects and OCDlike behaviours in Sapap3-mutant mice. Nature. 2007;448:894-900.

94.• Burguiere E, Monteiro P, Feng G, Graybiel AM. Optogenetic stimulation of lateral orbitofronto-striatal pathway suppresses compulsive behaviors. Science. 2013;340:1243-6. 
This paper describes the finding that optogenetic activation of IOFC projections to the CMS reverses a deficit in behavioral suppression in transgenic mice with an overgrooming phenotype.

95.•• Ahmari SE, Spellman T, Douglass NL, Kheirbek MA, Simpson HB, Deisseroth K, et al. Repeated corticostriatal stimulation generates persistent OCD-like behavior. Science. 2013;340:1234-9.

In this paper, the authors describe the finding that brief but repeated optogenetic activation of projections from $\mathrm{mOFC}$ to the VMS leads to the development of a persistent grooming phenotype in normal mice.

96.• Gremel CM, Costa RM. Orbitofrontal and striatal circuits dynamically encode the shift between goal-directed and habitual actions. Nat Commun. 2013;4:2264.

Using both traditional lesion techniques as well as optogenetics, the authors dissect the contribution of the DMS, DLS, and lOFC to goal-directed and habitual behaviors.
97. Diester I, Kaufman MT, Mogri M, Pashaie R, Goo W, Yizhar $\mathrm{O}$, et al. An optogenetic toolbox designed for primates. Nat Neurosci. 2011;14:387-97.

98. Desai M, Kahn I, Knoblich U, Bernstein J, Atallah $\mathrm{H}$, Yang A, et al. Mapping brain networks in awake mice using combined optical neural control and fMRI. J Neurophysiol. 2011;105:1393405.

99. Shipley FB, Clark CM, Alkema MJ, Leifer AM. Simultaneous optogenetic manipulation and calcium imaging in freely moving $\mathrm{C}$ elegans. Front Neural Circ. 2014;8:28.

100. Kent AR, Grill WM. Instrumentation to record evoked potentials for closed-loop control of deep brain stimulation. Conf Proc:... Ann Int Conf IEEE Eng Med Biol Soc IEEE Eng Med Biol Soc Ann Conf 2011;2011:6777-6780. 